Eur J Clin Chem Clin Biochem

1995; 33:221-224

(C) 1995 Walter de Gruyter \& Co.

Berlin · New York

\title{
In Vitro Effect of Dipyrone on Several Peroxidase Labelled Immunoassays
}

\author{
By Neus Gascón-Roche, Josefina Mora-Brugués, José Rodriguez-Espinosa, Mariano Cortés-Rius and \\ Francesc González-Sastre
}

Servei de Bioquímica, Hospital de la Santa creu i Sant Pau, Barcelona, Spain

(Received November 14, 1994/February 10, 1995)

Summary: We studied the in vitro effect of dipyrone on the determination of free triiodothyronine (free $T_{3}$ ), cortisol, progesterone, estradiol, carcinoembryonic antigen, human chorionic gonadotropin and $\alpha$-fetoprotein measured with an immunoenzyme assay based on enhanced luminescence that uses peroxidase as label. We found significant interference from dipyrone $(p<0.01)$ in the determination of all the analytes mentioned: for progesterone and estradiol the interference was present at high doses of dipyrone; for free $T_{3}$ and cortisol the minimum dipyrone concentration producing interference was $712 \mu \mathrm{mol} / 1$ and for carcinoembryonic antigen, human chorionic gonadotropin and $\alpha$-fetoprotein $44 \mu \mathrm{mol} / 1$. Dipyrone has an analytically and statistically significant interference effect on the determination of the mentioned analytes.

\section{Introduction}

Dipyrone (noramidopyrine methanesulfonate), one of the oldest synthetic pyrazolone derivatives, is an effective analgesic, antipyretic and anti-inflammatory drug (1). Due to its high solubility, it can be administered intravenously. Some of the secondary effects dipyrone can produce are: allergic reactions, skin eruptions and central nervous system alterations. In spite of its infrequent undesirable effect (agranulocytosis) (2), dipyrone is one of the most widely used analgesic drugs in Spain. It is mainly administered for the treatment of acute postsurgical or postraumatic pain and renal colic pain (3).

We have demonstrated the negative interference by dipyrone, in vitro and in vivo, in the determination of several biochemical analytes $(4,5)$. The common feature of the majority of these methods was a final reaction involving the enzyme peroxidase ${ }^{1}$ ).

Peroxidases consist of a wide group of enzymes with different functions. The longest and the best studied is

\footnotetext{
1) Peroxidase: EC 1.11.1.7
}

horseradish peroxidase (6) which is used in many immunoassay techniques.

The objective of this work is to examine the in vitro effect of dipyrone on several analytes measured by immunoassay techniques using peroxidase as a label.

\section{Materials and Methods}

Instrumentation

We measured the following analytes in serum: free $T_{3}$, cortisol, progesterone, estradiol, carcinoembryonic antigen, human chorionic gonadotropin and $\alpha$-fetoprotein. For all the measurements we used Amerlite reagents on the Amerlite Analyzer (Kodak Clinical Diagnostics Ltd, Amersham, UK).

\section{Procedures}

The determination of free $T_{3}$, cortisol, progesterone and estradiol is based on a competitive technique using enhanced luminescence. The method uses coated wells as solid phase and horseradish peroxidase as label. After removing the unbound fraction, the horseradish peroxidase activity of the bound conjugate is measured by an enhanced luminescence reaction obtained with the addition of the signal reagent that contains hydrogen peroxide as substrate, luminol as luminogen and a compound that enhances the intensity of light produced and prolongs its emission (the substituted phenol $p$-iodophenol). The amount of conjugate bound is inversely proportional to the concentration of analyte present in the sample. 
The Amerlite carcinoembryonic antigen, human chorionic gonadotropin and $\alpha$-fetoprotein assays are based on a non-competitive design involving a polyclonal antibody coated on to the wells and a horseradish peroxidase-conjugated monoclonal antibody. The horseradish peroxidase activity of the bound conjugate is measured by the enhanced luminescence reaction and the amount of conjugate bound is directly proportional to the concentration of analyte present in the sample.

Study in vitro

The possible interference of dipyrone on the different analytical methods was studied following the recommendations of the Sociedad Española de Química Clínica (7) and the guidelines of the National Committee for Clinical Laboratory Standards (8) for the detection and quantification of in vitro drug interference. We prepared a homogenous pool of fresh serum samples and we added dipyrone (Sigma, St. Louis, MO; cat. no. D-8890) to one half of this pool to a final concentration of $10 \mathrm{~g} / \mathrm{l}(28460 \mu \mathrm{mol} / \mathrm{l})$, ten-fold higher than the expected maximum concentration obtained after a standard dose of dipyrone, as suggested by Melvin R. Glick (9); the rest of the pool of serum containing different concentrations of the analytes studied was free from the interferent. Both pools were analyzed 15 times for each analyte. The outliers were eliminated by Dixon's criteria $(10,11)$. We tested the normality and homoscedasticity of the data by using the Agostino normality and Snedecor F tests, respectively, so that we could compare the means of both groups with results from the Mann-Whitney $U$ test. If the differences between means were significant $(p<0.01)$, the drug was considered to be an interferent.

To quantify the interference, we diluted the pooled sera containing $10 \mathrm{~g} / \mathrm{l}(28460 \mu \mathrm{mol} / \mathrm{l})$ of dipyrone with serum from the drug-free pool to obtain a series of samples containing dipyrone at 14230 , $7115,2846,1523,712,356,178,89$ and $44 \mu \mathrm{mol} / 1$. Five replicates of each sample were analyzed. Differences of mean concentrations of the analytes between the drug-free pool and the samples containing the interferent were evaluated for each method by using the Mann-Whitney U test.

\section{Results}

Table 1 shows the original concentrations determined for the serum samples we used in these studies and the minimum concentration of dipyrone that produced any interference with measurements of the different analytes.

Tab. 1 Original concentrations of the quantities and Minimum Dipyrone Concentration (MDC) producing interference in vitro

\begin{tabular}{|c|c|c|c|}
\hline Constituent & $\begin{array}{l}\text { Original } \\
\text { concentration }\end{array}$ & $\begin{array}{l}\mathrm{MDC} \\
(\mu \mathrm{mol} / \mathrm{l})\end{array}$ & $\mathrm{p}$ \\
\hline Free $T_{3}$ & $10.36 \mathrm{pmol} / 1$ & 712 & 0.0079 \\
\hline Cortisol & $529.8 \mathrm{nmol} / \mathrm{l}$ & 712 & 0.0079 \\
\hline Progesterone & $50.94 \mathrm{nmol} / 1$ & 14230 & 0.0079 \\
\hline Estradiol & $0.71 \mathrm{nmol} / 1$ & 2846 & 0.0079 \\
\hline $\begin{array}{l}\text { Carcinoembryonic } \\
\text { antigen }\end{array}$ & $256.5 \mu \mathrm{g} / \mathrm{l}$ & 44 & 0.0079 \\
\hline $\begin{array}{l}\text { Human chorionic } \\
\text { gonadotropin }\end{array}$ & $859.0 \mathrm{IU} / \mathrm{l}$ & 44 & 0.0079 \\
\hline$\alpha$-Fetoprotein & $312.7 \mathrm{kIU} / \mathrm{l}$ & 44 & 0.0079 \\
\hline
\end{tabular}

The results obtained from the quantification of the interference are represented by the interferographs, an easy format to present interference data obtained in the laboratory (9): the average assay value for each specimen was calculated as a fraction of the original concentration (without interferent); by plotting these fractions vs the concentration of potential interferant added, we drew these graphs (figs. 1-7).

\section{Discussion}

The main goal of an interference study is to determine if interference is present, and if so, to distinguish between a statistically significant effect and clinically significant interference. Lastly, the establishment of the type of interference offers a clearer understanding of its chemical nature.

The results from the Mann-Whitney $U$ test obtained in the studies in vitro were consistent with the presence of significant interference $(p<0.01)$ by dipyrone for all the analytes listed in tab. 1. It is important to note that the interference produced by dipyrone on the determination of progesterone and estradiol (at concentrations shown in table 1) is present only at dipyrone concentrations higher than the maximum concentration obtained after a standard dipyrone dose. For the rest of the quantities studied, the minimum dipyrone concentrations producing interference ranged from 44 to $712 \mu \mathrm{mol} / \mathrm{l}$. This range includes dipyrone concentrations that can be observed in a patient after a standard dipyrone dose (1).

The interference is positive or negative depending on the type of technology studied: positive interference in competitive methods and negative interference in noncompetitive ones. Dipyrone therefore interferes positively with the determination of free $T_{3}$, cortisol, estradiol and progesterone and negatively on the measurement of carcinoembryonic antigen, human chorionic gonadotropin and $\alpha$-fetoprotein.

From this data we demonstrate that dipyrone has an anàlytically and statistically significant in vitro interference on the concentration of several analytes determined by peroxidase labelled immunoassays. This interference is produced through a direct and specific inhibitory effect on the enzyme label since dipyrone can cause a varying loss of activity in peroxidase (12). We conclude that clinical laboratory data obtained by these methods in patients taking dipyrone should be carefully evaluated. 


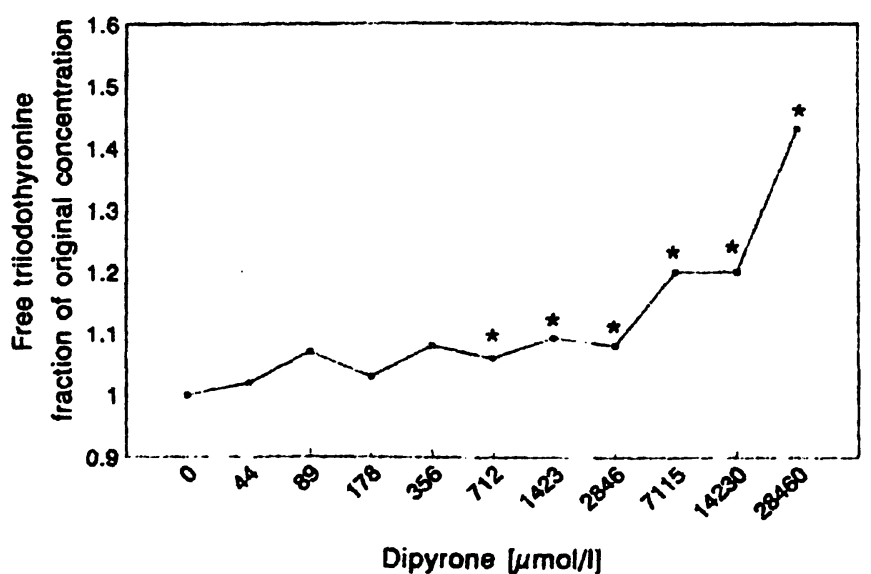

Fig. 1 Interferograph for free $T_{3}$

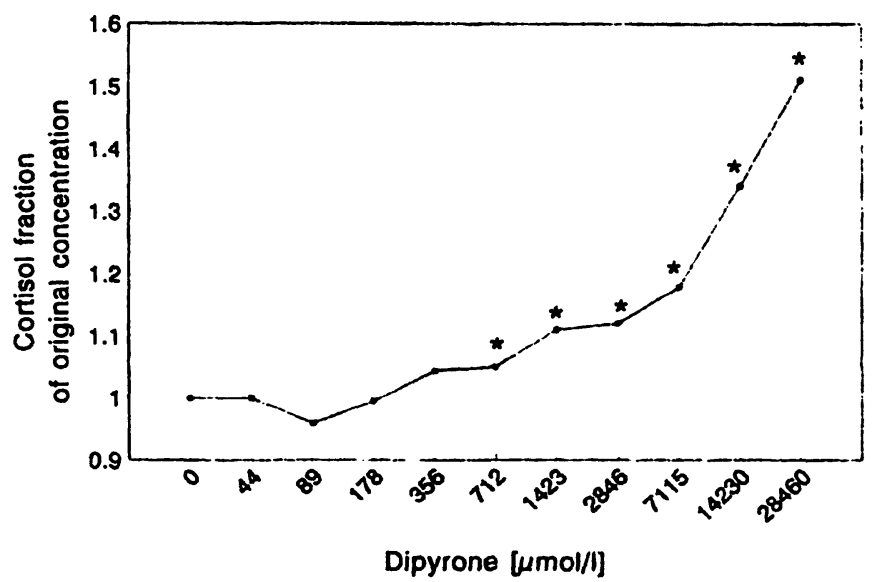

Fig. 2 Interferograph for cortisol

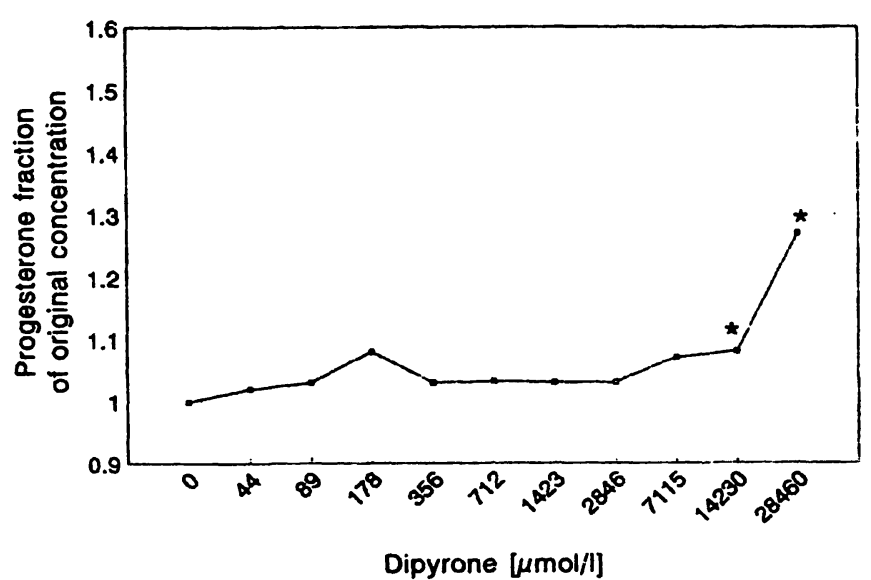

Fig. 3 Interferograph for progesterone

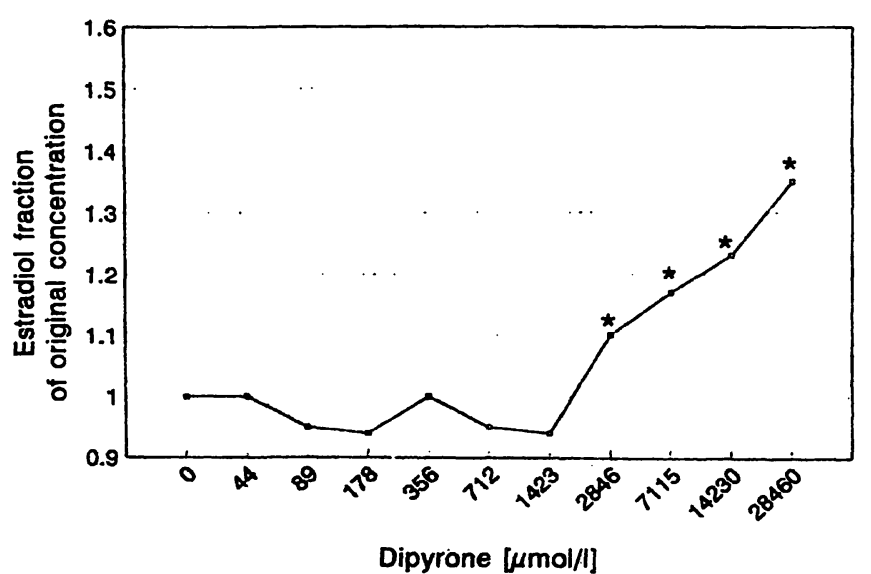

Fig. 4 Interferograph for estradiol

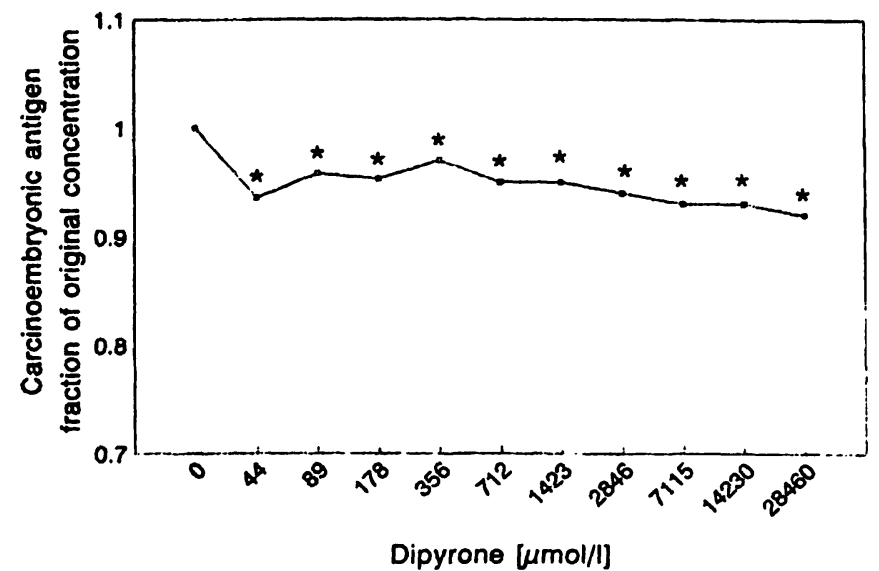

Fig. 5 Interferograph for carcinoembryonic antigen

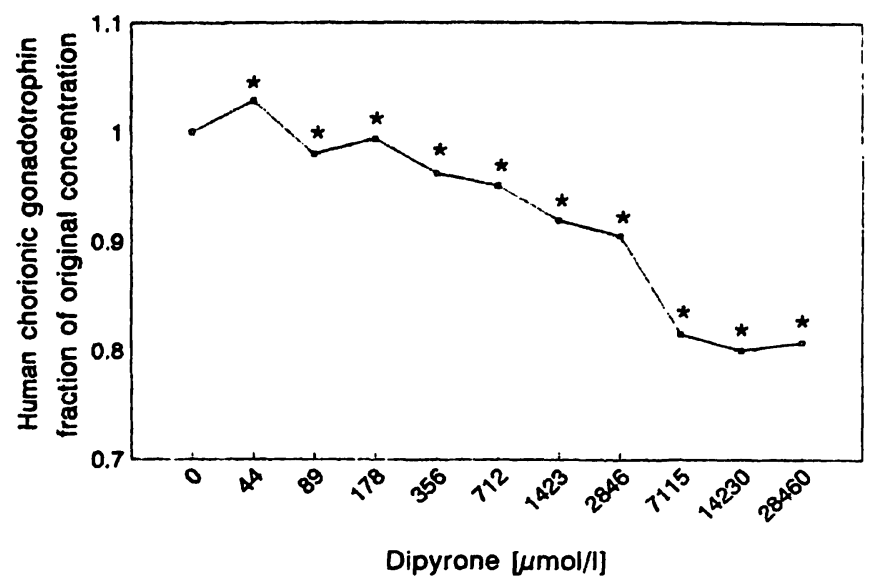

Fig. 6 Interferograph for human chorionic gonadotropin

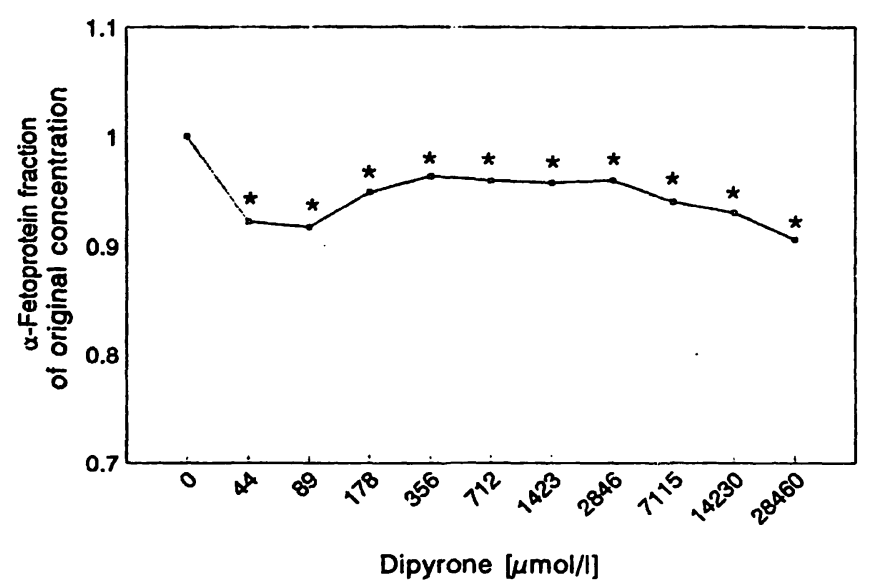

Fig. 7 Interferograph for $\alpha$-fetoprotein

Figs. 1-7

The starlets indicate significant $(p<0.01)$ interference 


\section{References}

1. Volz M, Kellner H-M. Kinetics and metabolism of pyrazolones (propyphenazone, aminopyrine and dipyrone). $\mathrm{Br} \mathrm{J}$ Clin Pharmac 1980; 10:299S-308S.

2. The International Agranulocytosis and Aplastic Anemia Study. Risk of agranulocytosis and aplastic anemia. A first report of the relation to drug use with special reference to analgesics. $J$ Am Med Assoc 1986; 256:1749-57.

3. Lloret J. Muñoz J, Monmany J, Puig X, Bonastre M, Brau J, et al. Treatment of renal colic with dipyrone. Curr Ther Res 1987; 42:1119-28.

4. Gascón N, Martínez-Brú C, Márquez M, Mercé J, Cortés M. Interférence du dipyrone dans la detérmination enzymatique de la créatinine avec un Kodak Ektachem. Ann Biol Clin 1992; 50:355.

5. Gascón N, Otal C, Martínez-Brú C, Mercé J, Cortés M, Arcelus $\mathrm{R}$, et al. Dipyrone interference on several common biochemical tests. Clin Chem 1993; 39:1033-6.

6. Pütter J. Peroxidases. In: Bergmeyer HU, editor. Principles of enzymatic analysis. Weinheim, New York: Verlag Chemie, 1978:685-90.

7. Comisión efectos de los medicamentos en química clínica. Documento B: protocolo para la valoración in vitro de interferencias por medicamentos. Quim Clín 1992; 11:449-52.
8. National Committee for Clinical Laboratory Standards. Interference testing in clinical chemistry; proposed guideline. NCCLS Publication 1986; EP7-P. Villnova, PA: NCCLS.

9. Glick MR, Ryder KW, Jackson SA. Graphical comparisons of interferences in clinical chemistry instrumentation. Clin Chem 1986; 32:470-5.

10. Dixon, WJ. Analysis of extreme values. Ann Math Statist 1950; 21:488-506.

11. Barnett V, Lewis T. Outliers in statistical data. 2nd ed. New York: John Wiley and Sond, 1984.

12. Gascón N, Cortés M, Mercé J, Mora J, González Sastre F. Inhibition of peroxidase by dipyrone. Calculation of the Michaelis constant $\left(\mathrm{K}_{\mathrm{m}}\right)$. [abstract] Clin Biochem Revs 1993; 14:242.

Neus Gascón-Roche

Servei de Bioquimica

Hospital de la Santa Creu i Sant Pau

Avda. Pare Claret, 167

E-08025 Barcelona

Spain 